\title{
Transanal total mesorectal excision with triangle rules: a road map to prevent injuries
}

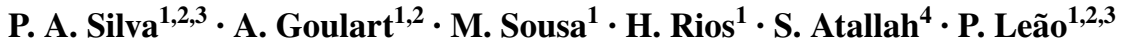

Received: 14 July 2017 / Accepted: 19 September 2017 / Published online: 24 October 2017

(C) Springer International Publishing AG 2017

Transanal total mesorectal excision (TaTME) is presented as a promising new surgical technique for the approach to the distal rectum, providing the ability to perform a highquality resection, with technical advantages over the laparoscopic approach [1]. However, the new surgical anatomy of the TaTME resection complicates surgical understanding and increases the risks of inadvertent injuries to important anatomic structures [2]. Detailed technical reports and stepwise description are necessary, to facilitate the surgeon's familiarization with this new technique and to reduce the learning curve in a safe manner. This video describes, in both sexes, the TaTME approach highlighting the unique anatomic structures and pitfalls, organized into four virtual triangles (anterior, posterior and two laterals) defining a road map of anatomic landmarks [3].

We report the case of a 47-year-old woman complaining of blood loss per rectum. A standard workup which included digital rectal examination, carcinoembryonic antigen,

P. A. Silva and A. Goulart have contributed equally to this work.

Electronic supplementary material The online version of this article (doi:10.1007/s10151-017-1698-5) contains supplementary material, which is available to authorized users.

\section{P. Leão}

pedroleao@ecsaude.uminho.pt

1 Department of General Surgery, Braga Hospital, Apartado 2241, 4701-965 Braga, Portugal

2 Life and Health Science Research Institute (ICVS), School of Medicine, University of Minho, Braga, Portugal

3 ICVS/3B's - PT Government Associate Laboratory, Braga/Guimarães, Portugal

4 Center for Colon and Rectal Surgery, Florida Hospital, Winter Park, FL, USA computed tomography scan of the chest and abdomen, pelvic magnetic resonance imaging scan and endorectal ultrasound identified a lesion at $3 \mathrm{~cm}$ from the anal verge, T2/T3a, and a lesion at $15 \mathrm{~cm}, \mathrm{~T} 2 \mathrm{~N} 0$, with no evidence of distant metastases. A biopsy was taken and histology revealed an adenocarcinoma. After a multidisciplinary oncology consultation, the patient had neoadjuvant chemoradiation followed by surgery.

Circumferential rectal dissection was initiated at the level of the anorectal ring and proceeded posteriorly and cranially in the avascular presacral plane, between the fascia propria of the rectum (enveloping the mesorectum and rectum) and presacral fascia (covering the presacral vein over the sacrum). In the beginning, the dissection needs to be in a downward direction, in order to avoid rectal perforation and respect the presacral plane. This posterior dissection can be more difficult because the posterior slope can be quite steep in some patients, so in these cases the sequence of TaTME does not need to be uniform and can be initiated by the anterior dissection. At the third and fourth sacral vertebra, the Waldeyer fascia is dissected, leading to a superior portion of the retrorectal compartment. The failure to recognize and divide it may result in perforation of the rectum or hemorrhage from the presacral venous plexus. In addition, full mobilization of the rectum is not possible unless the rectosacral fascia is divided. At the level of the sacral promontory, the mesorectum becomes a thick envelope mainly in the dorsolateral position containing perirectal fat, the superior rectal vessels, lymphatic tissue and nerve structures. Usually, the rectal fascia surrounding the mesorectum adheres to the inferior hypogastric plexus (IHP). If careful dissection is not performed, the IHP could be injured and this could lead to postoperative autonomic dysfunction [4].

The dissection is extended to the right and to the left of the rectum with the section of the lateral ligament and division of the middle rectal artery, if needed. The lateral ligament 
of the rectum consists of a broad band of dense connective tissues, which passes outward from the lateral walls of the rectum toward the base of the bladder at the point where the ureters terminate. This ligament appears lateral to the midrectum after dissection on the anterior and posterior sides of the rectum is completed. The lateral ligament is an extension of the mesorectum, anchoring it to the endopelvic fascia. It is divided into two segments by the IHP inside it and the visceral endopelvic fascia around it. The lateral ligament is a pathway of blood vessels and nerve fibers toward the rectum and lymphatic vessels from the lower rectum toward the iliac lymph nodes. Therefore, the lateral ligament plays a critical role in surgery for lower rectal cancer in two respects: the anatomic extent of resection for curing rectal cancer and the preservation of sexual function [5].

After extending the dissection posterolaterally, the dissection of the anterior mesorectum was performed to achieve circumferential rectal mobilization toward the peritoneal reflection. This is the unique triangle with the relevant anatomic differences between men and women. In men, the anterior part of the rectum is dissected from the seminal vesicle by incision of Denonvilliers' fascia. This membrane should be dissected at its posterior surface, to avoid injury of the bundles of Walsh, running from the 10 o'clock and 2 o'clock directions. However, for anteriorly located tumors, the anterior dissection may be necessary to achieve a negative margin. Even though the dissection is performed anteriorly, the excellent views achieved with TaTME can prevent injury to the neurovascular bundles of Walsh. At the level of the junction of the prostate and the seminal vesicles, the anterolateral dissection can facilitate the identification of the neurovascular bundles of Walsh because of the exposure of small vessels penetrating the prostate (the prostatic capsular artery and veins). Injury to the membranous (pre-prostatic) urethra can occur during very distal anterior dissection.

In women, the bundles of Walsh are identified at the level of junction of the lower and middle thirds of the vagina. The identification of the rectovaginal fascia also prevents injuries at the level of the bundles of Walsh. As a part of vaginal fascial structures, the rectovaginal fascia surrounds the posterior and lateral wall of the vagina and it could not be seen when the anterior rectal wall was dissected via an abdominal approach. Dissection in front of this fascia would destroy the integrity of the vaginal fascia and would damage the neurovascular bundle. Thus, dissection behind the rectovaginal ligament and in front of the fascia propria of the rectum should be more effective in preventing complications of rectal cancer excision, such as sexual dysfunction. The vaginal wall should be dissected carefully and sufficiently from the rectum, to prevent injury to the vagina and postoperative iatrogenic rectovaginal fistula. This dissection can be performed with the help of a uterine manipulator and digital examination. This possibility of digital control of the anterior dissection makes the female patient the best for beginners in this approach.

The peritoneal cavity was entered via a transabdominal approach with mesosigmoid and left paracolic gutter dissection and ligation of the inferior mesenteric vessels. Specimen extraction and anastomosis were performed transanally. A protective ileostomy was made and closed 4 weeks after discharge.

Operative time was $260 \mathrm{~min}$, and blood loss was estimated to be $20 \mathrm{ml}$.

On the third postoperative day, the patient presented a febrile peak. Screening for sepsis was negative. She was discharged on the eighth postoperative day.

The cancer was staged as ypT2G1N0R0 (0/16 lymph nodes). The tumor size was $1 \times 0.3 \mathrm{~cm}$, and histologically, the lesion was consistent with low-grade, well-differentiated, adenocarcinoma of the rectum with grade 1 tumor regression according to the modified Dworak tumor regression grading system. The specimen had intact mesorectum with a smooth and regular surface. The proximal margin measured $11.5 \mathrm{~cm}$, the distal margin measured $3.5 \mathrm{~cm}$, while the circumferential margin was $1.5 \mathrm{~cm}$.

\section{Compliance with ethical standards}

Conflict of interest The authors declare that they have no conflict of interest.

Ethical approval This video forum does not contain studies with the patient. The surgery was documented, with proper informed consent ant with prior authorization of the patient, it was edited for publication. This study is in accordance with the ethical standards.

Informed consent Informed consent was obtained in accordance with the standards set forth by hospital regulations.

\section{References}

1. Lacy AM, Rattner DW, Adelsdorfer C et al (2013) Transanal natural orifice transluminal endoscopic surgery (NOTES) rectal resection: "down-to-up" total mesorectal excision (TME)-short-term outcomes in the first 20 cases. Surg Endosc 27:3165-3172

2. Chouillard E, Regnier A, Vitte R-L et al (2016) Transanal NOTES total mesorectal excision (TME) in patients with rectal cancer: is anatomy better preserved? Tech Coloproctol 20(8):537-544

3. Leão P, Goulart A, Veiga C et al (2015) Transanal total mesorectal excision: a pure NOTES approach for selected patients. Tech Coloproctol 19:541-549

4. Aigner F, Hörmann R, Fritsch H, TAMIS TME Collaboration Group et al (2015) Anatomical considerations for transanal minimal- invasive surgery: the caudal to cephalic approach. Colorectal Dis 17(2):O47-O53

5. Wang GJ, Gao CF, Wei D, Wang C, Meng WJ (2010) Anatomy of the lateral ligaments of the rectum: a controversial point of view. World J Gastroenterol 16(43):5411-5415 